\title{
Addendum
}

\section{A Mathematical Classification of the One-Dimensional Deterministic Cellular Automata}

Michel Dubois-Violette and Alain Rouet

Laboratoire de Physique Théorique et Hautes Energies, Université Pairs Sud, Batiment 311, F-91405 Orsay, France

Commun. Math. Phys. 112, 627-631 (1987)

We received the following letter from E. M. Coven:

"I write to bring to your attention the paper of G. A. Hedlund, Endomorphisms and automorphisms of the shift dynamical system, [Math. Syst. Theory 3,320-375 (1969)]. This excellent survey article deals with some of the work done on onedimensional deterministic cellular automata in the 1950's and 1960's, a time when neither the subject nor the term was in vogue.

In particular, the theorem in your recent paper on one-dimensional deterministic cellular automata [Commun. Math. Phys. 112, 527-631 (1987)] is Theorems 5.4 and 5.9 in Hedlund's paper."

E. M. Coven is perfectly right, although our methods are quite different. We thank him for this information and take this opportunity to draw the attention to the above quoted reference. 